\title{
Schizotypal traits are not related to multisensory integration or audiovisual speech perception
}

\author{
Anne-Marie Muller ${ }^{1,2}$, Tyler C. Dalal ${ }^{1,2}$, Ryan A. Stevenson ${ }^{1,2}$ \\ ${ }^{1}$ Department of Psychology, University of Western Ontario, London, ON, Canada \\ ${ }^{2}$ Brain and Mind Institute, University of Western Ontario, London, ON, Canada
}

Correspondence should be addressed to Ryan A. Stevenson, Western Interdisciplinary Research Building, 1151 Richmond St, London, Ontario, N6A 5B7, Canada. Email: rsteve28@uwo.ca 


\begin{abstract}
Multisensory integration, the binding of sensory information from different sensory modalities, may contribute to perceptual symptomatology in schizophrenia, including hallucinations and aberrant speech perception. Differences in multisensory integration and temporal processing, an important component of multisensory integration, are consistently found in schizophrenia. Evidence is emerging that these differences extend across the schizophrenia spectrum, including individuals in the general population with higher schizotypal traits. In the current study, we investigated the relationship between schizotypal traits and perceptual functioning, using audiovisual speech-in-noise, McGurk, and ternary synchrony judgment tasks. We measured schizotypal traits using the Schizotypal Personality Questionnaire (SPQ), hypothesizing that higher scores on Unusual Perceptual Experiences and Odd Speech subscales would be associated with decreased multisensory integration, increased susceptibility to distracting auditory speech, and less precise temporal processing. Surprisingly, neither subscales were associated with any of these measures. These results suggest that perceptual differences may not be present across the schizophrenia spectrum.
\end{abstract}

Keywords: multisensory integration, schizophrenia spectrum disorders, schizotypy, audiovisual, speech perception, distractibility, McGurk effect, ternary synchrony judgment task, speech-innoise task, schizophrenia 


\section{Introduction}

The integration of auditory and visual speech signals is essential for accurate perception of everyday audiovisual speech. These integration processes are especially important in noisy environments, in which one cannot rely solely on auditory signals, and the speaker's facial movements can be especially helpful (Ross, Saint-Amour, Leavitt, Javitt, \& Foxe, 2007). The process by which auditory and visual speech signals are integrated, multisensory integration, is highly complex, as the brain must decide from an overwhelming amount of sensory information which information occurred together and should be integrated, and which should not (Stevenson, Ghose, et al., 2014). Temporal processing plays a crucial role in multisensory integration, as temporal proximity of auditory and visual signals is a cue to bind, such that the closer together in time two signals are, the more likely they are to be integrated (Stein \& Meredith, 1993; Vroomen \& Keetels, 2010)

Investigators have begun to explore the possibility that impairments in both multisensory integration and temporal processing may be contributing to perceptual deficits found among individuals with schizophrenia. A number of theoretical frameworks of schizophrenia predict difficulties in multisensory integration. Some theories, such as the disconnection hypothesis, postulate that schizophrenia symptomatology is associated with decreased inter-region connectivity (Friston, Brown, Siemerkus, \& Stephan, 2016). Such inter-region connectivity between auditory and visual systems is necessary for multisensory integration, and thus this hypothesis may also predict atypical integration. Alternatively, theories such as the panmodal processing imprecision hypothesis directly postulate that schizophrenia symptomatology is derived in part from imprecise sensory processing across modalities (Javitt, Liederman, Cienfuegos, \& Shelley, 1999). 
In support of these theories, there have been numerous empirical findings of impaired temporal processing of sensory inputs in individuals with schizophrenia, both in the visual modality alone (Capa, Duval, Blaison, \& Giersch, 2014; de Boer-Schellekens, Stekelenburg, Maes, Van Gool, \& Vroomen, 2014; Giersch et al., 2009; Lalanne, Van Assche, Wang, \& Giersch, 2012; Schmidt, McFarland, Ahmed, McDonald, \& Elliott, 2011; Tenckhoff, Tost, \& Braus, 2002) and in audiovisual modalities together (Foucher, Lacambre, Pham, Giersch, \& Elliott, 2007; Martin, Giersch, Huron, \& Wassenhove, 2013; Noel, Stevenson, \& Wallace, 2018; Stevenson, Park, et al., 2017). Individuals with schizophrenia also have decreased audiovisual multisensory integration compared to controls (de Jong, Hodiamont, Van den Stock, \& de Gelder, 2009; Ross et al., 2007; Tseng et al., 2015; Williams, Light, Braff, \& Ramachandran, 2010) (Zhou et al., 2018). These deficits in multisensory integration are pronounced during speech perception, providing evidence for a logical link between multisensory integration and temporal processing impairments and symptoms of social communication problems in schizophrenia (Tseng et al., 2015).

As a result of impaired multisensory integration and temporal processing, individuals with schizophrenia gain less benefit from seeing visual speech stimuli in audiovisual speech in the context of noise (Ross, Saint-Amour, Leavitt, Molholm, et al., 2007). Individuals with schizophrenia also have impairments in unisensory auditory speech perception in the context of background speech noise (Hoffman, Rapaport, Mazure, \& Quinlan, 1999; Shedlack et al., 1997; Wu et al., 2012), particularly individuals with schizophrenia who experience auditory hallucinations (Hoffman et al., 1999; Lee, Chung, Yang, Kim, \& Suh, 2004). However, individuals with schizophrenia are also impaired in the perception of auditory words without background noise (Bull \& Venables, 1974; DeLisi et al., 1997; Shedlack et al., 1997; Titone \& 
Levy, 2004). These deficits appear to be present at early stages of auditory phoneme processing, as revealed by altered event-related potentials in response to phoneme changes (Kasai et al., 2002, 2003).

Individuals with schizophrenia are also impaired at ignoring distracting speech, resulting in poorer perception accuracy of target speech and increased perception of irrelevant, distracting speech (Moser, Cienfuegos, Barros, \& Javitt, 2001; Oltmanns \& Neale, 1975). Indeed, speech perception is more impaired when embedded in background speech than when embedded in white noise (Wu et al., 2012), and greater impairments in ignoring such distracting speech is related to severity of disorganized speech production (Moser et al., 2001). Additionally, a greater tendency to extract meaningful speech from incomprehensible overlapping background babble among individuals with prodromal psychosis symptoms is predictive of subsequent schizophrenia diagnosis (Hoffman et al., 2007). This greater susceptibility of individuals across the schizophrenia spectrum to attend to distracting auditory speech appears to be due to deficits either in the allocation of attention, in the available attentional resources (Bestelmeyer, 2012), or in sensory gating abilities (Mcdowd, Filion, Harris, \& Braff, 1993). Taken together, this evidence suggests that impaired multisensory integration and temporal processing, in combination with increased distractibility, may lead to impaired speech perception.

These three perceptual issues, impaired multisensory integration and temporal processing and increased distractibility, potentially also contribute to clinical symptoms such as auditory speech hallucinations (Stevenson, Park, et al., 2017). One of the leading theories explaining auditory verbal hallucinations suggests that they occur when individuals mistakenly interpret their own inner voice as external (Jones, 2010). Taken further, considering that most hallucinations experienced by individuals with schizophrenia are audiovisual (Lim et al., 2016; 
McCarthy-Jones et al., 2017), such hallucinations could arise from the erroneous binding of visual external cues with inner auditory speech (Jones \& Fernyhough, 2007). Such erroneous binding may arise from issues with temporal processing. With that said, while individuals with schizophrenia have less precise temporal processing than controls overall, within individuals with schizophrenia, those who had more severe hallucinations tended to have narrower temporal binding windows (Stevenson, Park, et al., 2017). Evidently, the role of temporal processing in contributing to schizophrenia symptoms may be more complex than expected. While narrower temporal binding windows are usually thought to be more beneficial as they reflect more precise multisensory temporal precision, wider windows may be adaptive for individuals with schizophrenia due to unreliable unisensory processing. Finally, increased distractibility to auditory speech may also contribute to this erroneous binding. Increased attention to irrelevant speech may result in integrating this irrelevant speech with what is seen visually. Considering that individuals with schizophrenia are slightly poorer lip-readers (Schonauer, Achtergarde, \& Reker, 1998), they may be more likely to take irrelevant auditory information and bind it mistakenly with visual facial speech information.

The current study aims to investigate the hypothesis that impaired multisensory integration and temporal processing, as well as increased distractibility, contribute to symptoms within the schizophrenia spectrum. We will investigate this hypothesis by measuring these perceptual processes in individuals in the general population with higher levels of schizotypal traits. These schizotypal traits, measured by various self-report questionnaires, are attenuated versions of symptoms found in schizophrenia, covering domains of unusual perceptual experiences, communication, social behaviour, delusion-like thoughts, and suspiciousness (Raine, 1991). Multiple lines of evidence have converged to support the idea of the 
schizophrenia spectrum, with similarities being found between individuals with schizotypal traits and schizophrenia in genetic, cognitive, perceptual, and neurobiological areas (Ettinger, Meyhöfer, Steffens, Wagner, \& Koutsouleris, 2014). Not only do individuals with higher levels of schizotypal traits show many similarities to individuals with schizophrenia, but they are also at higher risk of developing schizophrenia in the future (Kwapil, Gross, Silvia, \& BarrantesVidal, 2013). Investigating perceptual deficits within individuals with these lower-level traits can therefore be seen as a way of identifying cognitive biomarkers present across the spectrum that may be found in individuals who are prodromal for schizophrenia, in order to understand how to better predict the development of schizophrenia and begin treatment earlier for these individuals. As mentioned earlier, some of these potential cognitive biomarkers, which we will investigate in the current study, are in the areas of multisensory integration and temporal processing, as well as speech perception and auditory distractibility, all of which appear to be perceptual commonalities across the schizophrenia spectrum. Early evidence suggests that individuals with higher levels of schizotypal traits have poorer temporal processing in tactile-proprioceptive (Ferri, Ambrosini, \& Costantini, 2016) and audio-tactile (Ferri et al., 2017) domains, suggesting wider temporal binding windows. Additionally, those with higher schizotypy have also been found to have increased perception of the audiovisual double-flash (fission) illusion (Ferri, Venskus, Fotia, \& Cooke, 2018), which also suggests wider temporal binding windows in these individuals. Additionally, individuals with higher schizotypy have stronger responses to visualtactile (rubber-hand and Barbie doll) illusions, demonstrating altered multisensory integration (Asai, Mao, Sugimori, \& Tanno, 2011; Germine, Leigh, Cohen, \& Lee, 2013; Van Doorn, De Foe, Wood, Wagstaff, \& Hohwy, 2018). These individuals are also more easily distracted by 
auditory speech (Marsh, Vachon, \& Sörqvist, 2017) and visual non-speech stimuli (BraunsteinBercovitz \& Lubow, 1998).

We aimed to replicate these findings of altered audiovisual temporal processing, multisensory integration, and auditory speech distractibility, as well as to investigate for the first time whether schizotypy is associated with poorer audiovisual speech perception. Here, in three experiments, we assessed the relationship between schizotypal traits and measures of audiovisual speech perception, auditory speech distractibility, multisensory integration, and temporal processing. All participants completed all three experiments, as well as the Schizotypal Personality Questionnaire (SPQ; Raine, 1991), a measure of schizotypal traits. Experiment 1 used a speech-in-noise task to assess auditory, visual, and audiovisual speech perception, as well as multisensory gain and susceptibility to distracting auditory speech relative to schizotypal traits. In Experiment 2, multisensory integration was directly tested using the McGurk Effect, and was related to schizotypal traits. Finally, in Experiment 3, the precision of multisensory temporal processing of speech stimuli was measured and related to schizotypal traits. The hypotheses, methods, and analyses of the present study were pre-registered within the Open Science Framework (link to the locked pre-registration: https://osf.io/f2vsz/?view_only=ccd886cd0d26462b96593f8c0e4b498e). Within this preregistration, it was hypothesized that reduced audiovisual speech perception, multisensory integration and temporal processing across tasks would be associated with higher levels of schizotypal traits, specifically Unusual Perceptual Experiences and Odd Speech.

\section{General Materials and Methods}

\subsection{Participants}


Ninety-nine $(70$ female, mean age $=18.10$ years old, $\mathrm{SD}=0.92$, range $=17-22)$ from Western University completed the current experiment. 19 additional participants were excluded (eight did not complete the questionnaires, seven did not complete all of the behavioural measures, and four did not follow task directions). Experimental protocols were approved by Western University's Non-medical Research Ethics Board. All individuals self-reported normal hearing and normal or corrected-to-normal vision. All participants reported that English was the first language they learned. Participants were recruited via the undergraduate research participation pool. All participants completed all three experiments, including the speech ternary synchrony judgment (SJ3) task, both multisensory and unisensory versions of the McGurk task, the speech-in-noise (SiN) task, and the Schizotypal Personality Questionnaire (SPQ; Raine, 1991).

\subsection{Schizotypal Traits}

The Schizotypal Personality Questionnaire (SPQ; Raine, 1991) is a 74-item questionnaire frequently used in the general population. Each item requires either a "Yes" or "No" answer, with the total score summing up "Yes" responses. The items of the SPQ can be reliably broken down into nine subscales: Ideas of Reference, Excessive Social Anxiety, Odd Beliefs or Magical Thinking, Unusual Perceptual Experiences, Odd or Eccentric Behavior, No Close Friends, Odd Speech, Constricted Affect, and Suspiciousness. A widely-replicated three-factor model of the SPQ groups the nine subscales into three factors that match the three areas impacted in both schizophrenia and schizotypal personality disorder: Cognitive-Perceptual, Disorganized, and Interpersonal (Badcock \& Dragović, 2006; Chen, Hsiao, \& Lin, 1997; Fossati, Raine, Carretta, Leonardi, \& Maffei, 2003; Raine, Lencz, Scerbo, \& Kim, 1994; Reynolds, Raine, Mellingen, Venables, \& Mednick, 2000; Wuthrich \& Bates, 2006). High sampling validity has been reported 
for the SPQ, as well as high internal reliability (0.91), test-retest reliability (0.82), convergent validity (0.59 to 0.81$)$, discriminant validity, and criterion validity $(0.63,0.68)$ (Raine, 1991$)$.

Overall SPQ total, factor, and subscale scores were calculated for each participant (Figure 1). Using the $10 \%$ high and low cut-offs reported for the original validation of the SPQ of 41 and 12, respectively (Raine, 1991), seven individuals, or $7.07 \%$, met the high cut-off, and 20 individuals, or $20.20 \%$, met the low cut-off. As such, the current sample filled a wide range of the scale.
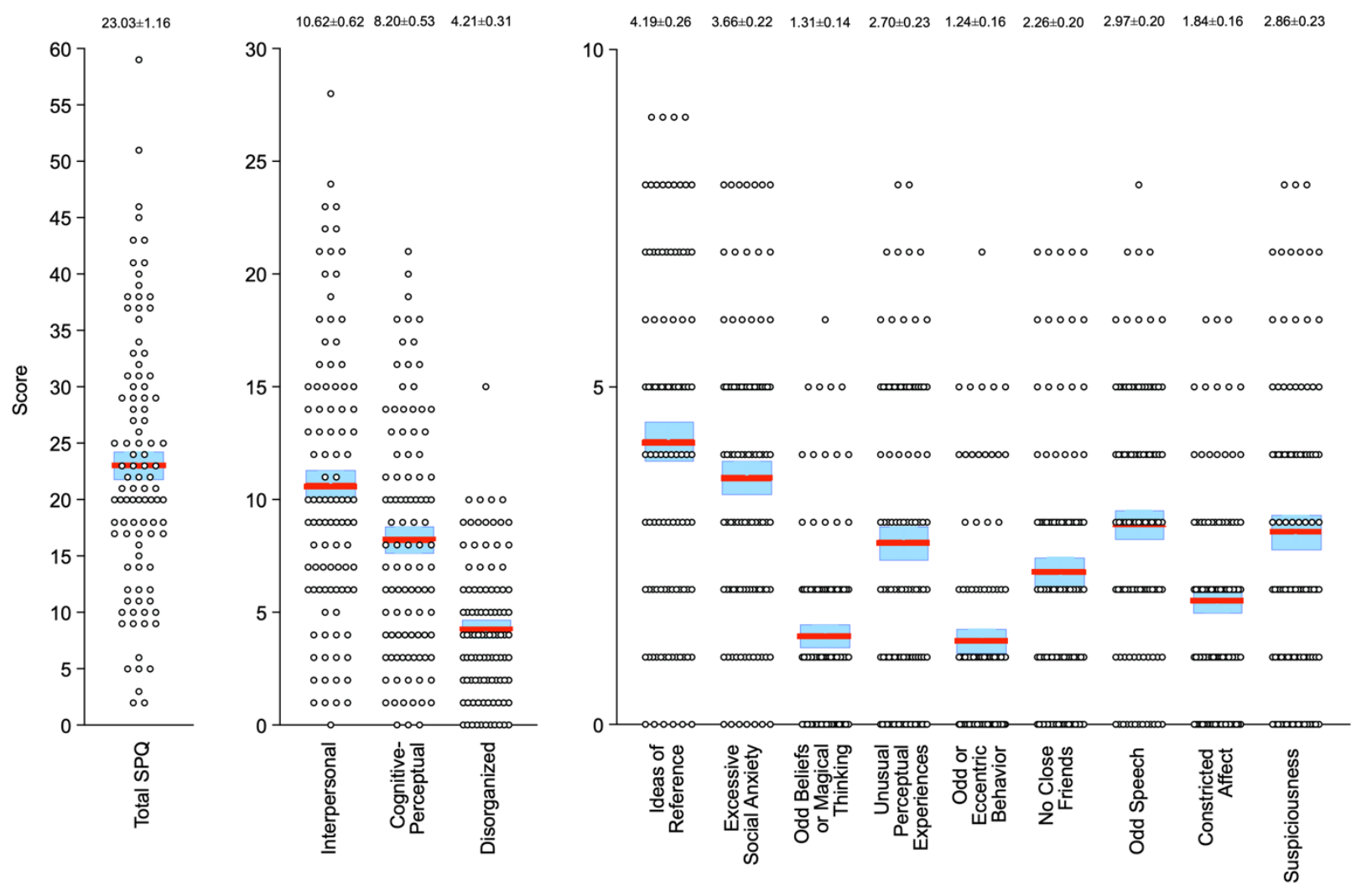

Figure 1. Overall SPQ total, factor, and subscale scores for each participant $(N=99)$.

Note. Red lines indicate mean and blue error bars indicate standard error. Red lines indicate group means, and blue indicates standard error. Values above each cluster indicate mean \pm standard error. Maximum possible scores for SPQ total, factor, and subscales are, in order: 74, $33,25,16,9,8,7,9,7,9,9,8,8$. 


\subsection{General Procedures}

The SPQ was completed online through Qualtrics prior to each study visit. For Experiments 1, 2, and 3, participants sat approximately $60 \mathrm{~cm}$ from the monitor, in a sound- and light-controlled room. A researcher sitting next to the participant monitored the participant to make sure his/her eyes remained on the screen. All stimuli throughout Experiments 1, 2, and 3 were presented using MATLAB 2018a (MATHWORKS Inc., Natick, MA) software with the Psychophysics Toolbox extensions (Brainard, 1997; Pelli, 1997). Audio stimuli were presented binaurally through BOSE QuietComfort 35 noise-cancelling headphones.

\section{Experiment 1}

\subsection{Rationale and Hypotheses}

Experiment 1 used a speech-in-noise task (Stevenson et al., 2015; Stevenson et al., 2011; Stevenson et al., 2010; Stevenson and James, 2009; Stevenson et al., 2009), in which individuals must identify words in the presence of noisy background speech, to measure audiovisual speech perception. Using a modification of the speech-in-noise task, in which additional auditory distractor words were added, susceptibility to these distractor words were assessed. The purpose of this manipulation was to test the hypothesis that poorer abilities to tune out distracting auditory information, resulting in unrelated auditory information being mistakenly bound with visual information, could contribute to auditory hallucinations among individuals across the schizophrenia spectrum. The speech-in-noise task also allowed for the measurement of multisensory integration by comparing speech perception in unisensory and multisensory conditions. Gains in speech perception between unisensory and multisensory conditions indicated multisensory integration. 
It was hypothesized that two subscales within the SPQ would be associated with the speech-in-noise task: Unusual Perceptual Experiences and Odd Speech. We hypothesized that individuals with higher levels of these perceptual- and speech-based schizotypal traits would have reduced multisensory integration, showing less benefit from multisensory input relative to unisensory input in the speech-in-noise task. We also expected that individuals with higher schizotypy would demonstrate reduced audiovisual speech perception, and increased susceptibility to distracting auditory speech. We expected this increased susceptibility to be the case especially for the "Different Time" condition, in which the distracter word preceded the target word by $250 \mathrm{~ms}$.

\subsection{Materials and Methods}

\subsubsection{Stimuli}

Stimuli for the speech-in-noise task included audiovisual (AV) recordings of a female speaker saying 144 triphonemic nouns. Stimuli were selected from a previously published stimulus set, The Hoosier Audiovisual Multi-talker Database (Sheffert et al., 1996). All stimuli were spoken by speaker F1. The stimuli selected were monosyllabic English words that were matched across sets for accuracy on both visual-only and audio-only recognition (Lachs and Hernandez, 1998), and were also matched across sets in lexical neighborhood density (Luce and Pisoni, 1998; Sheffert et al., 1996). Audio signal levels were measured as root mean square (RMS) contrast and equated across all words. All words lasted $2 \mathrm{~s}$ and included all prearticulatory gestures. Visual stimuli were grayscale and square, spanning $9.9 \mathrm{~cm}$ per side or $9.43^{\circ}$ of visual angle. This set of single words has been used successfully in previous studies of multisensory integration (e.g. Stevenson et al., 2015). 
All presentations included 8-channel multitalker babble at $66 \mathrm{~dB}$ SPL. The presentation of auditory babble presentation began $500 \mathrm{~ms}$ prior to the beginning of the word and ended 500 ms following the end of the word. The RMS of the auditory babble was linearly ramped up and down, respectively, during the pre- and post-stimulus $500 \mathrm{~ms}$ periods, and was presented with the first and last frames of the visual word, respectively. Auditory stimuli were presented at two signal-to-noise ratios (SNR), $54 \mathrm{~dB}$ (-12 dB SPL SNR) or $66 \mathrm{~dB}$ (0 dB SPL SNR).

\subsubsection{Procedures}

Each participant was presented with six separate runs of 24 single-word presentations consisting of a single condition, for a total of 144 words. Two of the conditions were unisensory, while the other four were audiovisual. All six conditions, including the visual-only condition, included auditory multitalker babble at $66 \mathrm{~dB}$ SPL. Three of the audiovisual conditions included a second, auditory-only distractor word in addition to the target word. One of these auditory words corresponded to the visual speaker (target), and one word did not (distractor). The visual word presentations were never manipulated.

The six conditions were:

1) Visual-only: visual speaker only, no auditory word presentation.

2) Auditory-only: auditory target words only, at $54 \mathrm{~dB}$ SPL; blank screen, no visual word presentation.

3) Audiovisual: visual and auditory presentations of the same word at the same time, at 54 dB SPL.

4) Same time audiovisual: auditory target and distractor words presented at same time as visual target word. Both target and distractor words at $54 \mathrm{~dB}$ SPL. 
5) Different volume audiovisual: auditory target and distractor words presented at same time as visual target word, with false word $12 \mathrm{~dB}$ SPL louder. Distractor words at $66 \mathrm{~dB}$ SPL; target words at $54 \mathrm{~dB}$ SPL.

6) Different time audiovisual: auditory target and distractor words presented at different times, with distractor word beginning $250 \mathrm{~ms}$ before presentation of visual and auditory target word. Both target and distractor words at $54 \mathrm{~dB}$ SPL.

Experimental procedures were identical for all runs. Participants were instructed to attend to the speaker at all times, and to report the word they perceived by typing "What the speaker said". The experimenter verbally confirmed the participant's report to correct for spelling errors and ambiguous pronunciation, and then the next word was presented. No time limit was given for participant responses. Each run lasted approximately 5 minutes, and run orders and condition-to-word-list pairings (including distractor word lists) were randomized across participants. No words were repeated.

\subsubsection{Analysis}

As done previously (Stevenson et al., 2015; Stevenson, Segers, et al., 2017), responses were scored at both the whole-word level and at the phoneme level. Whole words were scored as correct only if the entire word reported was correct. Each tri-phonemeic word was also scored on the proportion of phonemes that were perceived correctly. When distractor words were present, scores were tabulated for both. Word and phoneme accuracies were calculated as the average score across all trials for each condition, as was distractor susceptibility.

Multisensory gain was calculated by comparing accuracy scores in audiovisual trials relative to the predicted audiovisual accuracy based on the unisensory component accuracies assuming independence, using the following equation (Stevenson et al., 2015): 


$$
p A V=p(A)+p(V)-[p(A) * p(V)],
$$

where pAV represents a null hypothesis of the response to audiovisual presentations if the auditory and visual information are processed independently, and where $p(A)$ and $p(V)$ represent response accuracy to auditory- and visual-only presentations, respectively. Absolute increase was calculated as:

$$
\text { observed } A V \text { accuracy - pAV accuracy, }
$$

while proportion increase was calculated as:

$$
\frac{\text { observed } A V \text { accuracy }-p A V \text { accuracy }}{1-p A V \text { accuracy }}
$$

Pearson correlations were then conducted between distractor word and phoneme susceptibility and SPQ scores, as well as between multisensory gain and SPQ scores.

\subsection{Results}

The proportion of word and phoneme accuracy for all six conditions of the speech-innoise task are shown for each individual in Figures 2 and 3. Multisensory gain was significantly greater than zero in all instances, including word accuracy measured by absolute $(t(98)=14.55$, $\mathrm{p}<0.001, \mathrm{~d}=1.46)$ and proportional $(t(98)=14.65, \mathrm{p}<0.001, \mathrm{~d}=1.47)$ gain, and phoneme accuracy measured by absolute $(t(98)=7.05, \mathrm{p}<0.001, \mathrm{~d}=0.71)$ and proportional $(t(98)=6.12$, $\mathrm{p}<0.001, \mathrm{~d}=0.62)$ gain. 

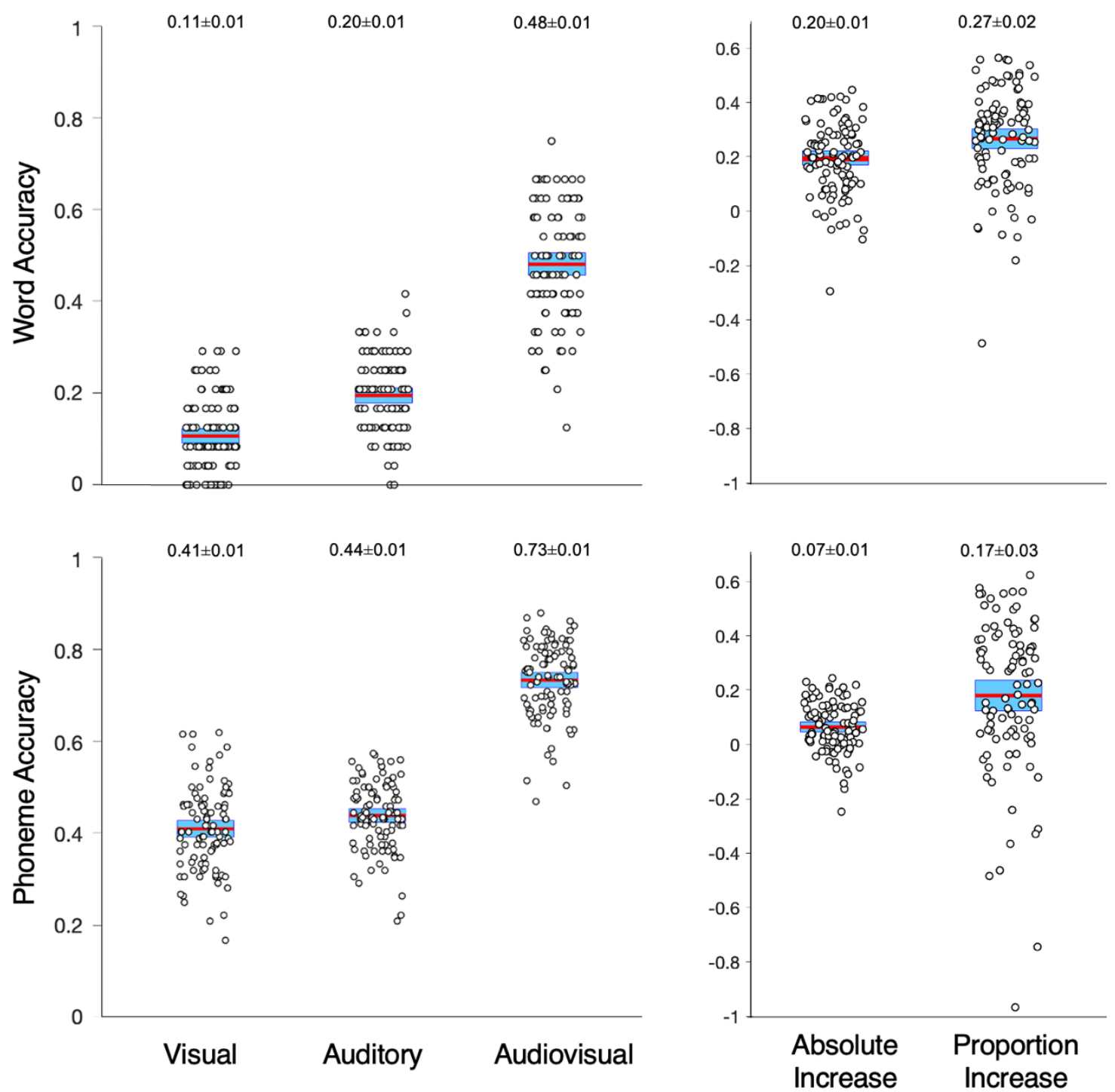

Audiovisual Gain

Figure 2. Proportion of word and phoneme accuracy for visual, auditory, and audiovisual conditions of the speech-in-noise task, as well as audiovisual gain.

Note. Red lines indicate group means, and blue indicates standard error. Values above each cluster indicate mean \pm standard error. 

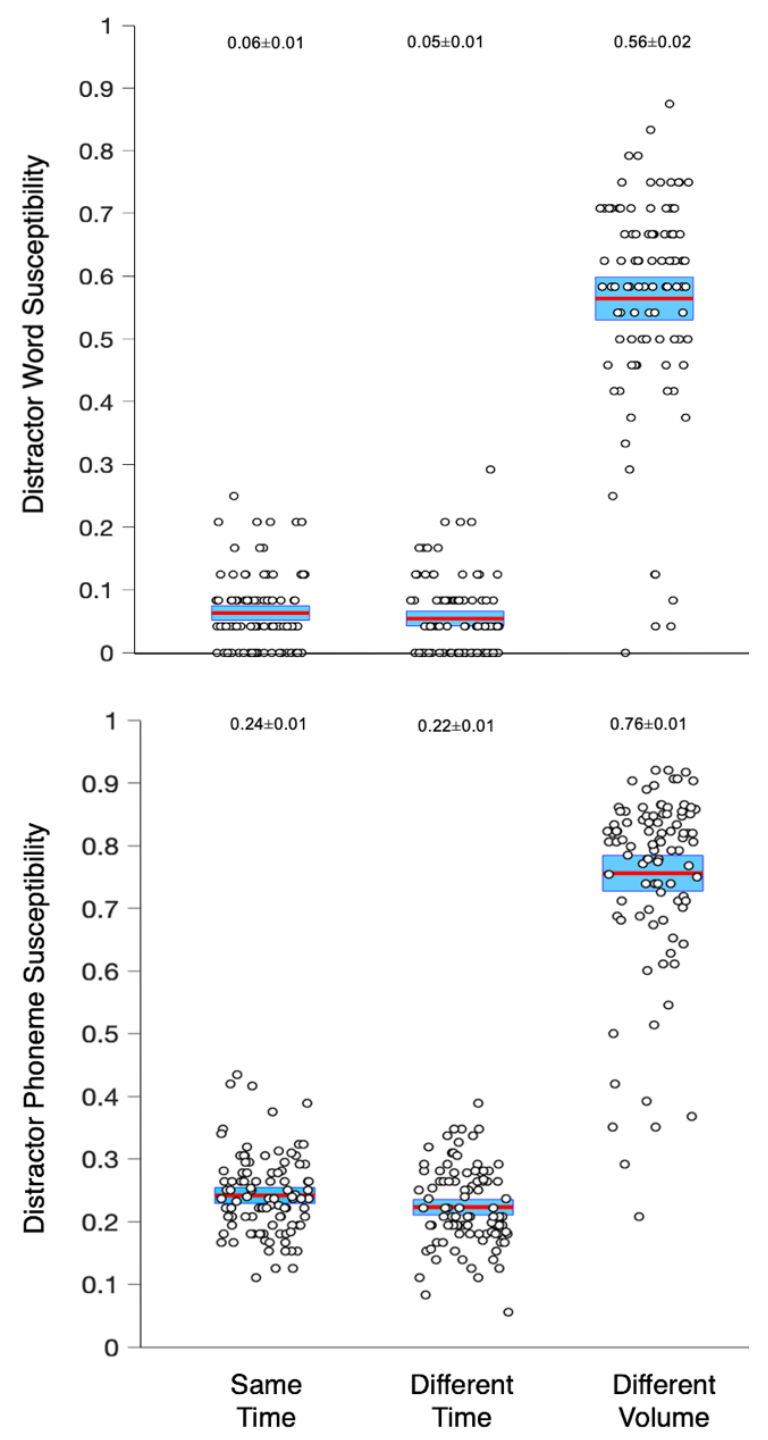

Figure 3. Proportion of word and phoneme accuracy for Same Time, Different Time, and Different Volume conditions of the speech-in-noise task.

Note. Red lines indicate group means, and blue indicates standard error. Values above each cluster indicate mean \pm standard error.

\subsubsection{Relating Speech-in-Noise Task and Schizotypal Traits}

Pearson correlations were conducted between word and phoneme accuracy and SPQ scores, as well as between multisensory gain and SPQ scores. Target word and phoneme accuracy were expected a priori to negatively correlate with Unusual Perceptual Experiences 
and Odd Speech. Multisensory gain was expected a priori to negatively correlate with two subscales of the SPQ: Unusual Perceptual Experiences and Odd Speech. Distractor word and phoneme susceptibility were expected a priori to positively correlate with Unusual Perceptual Experiences and Odd Speech.

In the audiovisual condition, target phoneme accuracy was not significantly correlated with scores on the Unusual Perceptual Experiences subscale, nor was target word accuracy. Similarly, in the audiovisual condition, target phoneme accuracy was not significantly correlated with scores on the Odd Speech subscale, nor was target word accuracy (Table 1). As all Bayes factors were below 0.160 , this provides substantial evidence (according to Jeffreys in Jarosz \& Wiley (2014)) in support of the null hypothesis that Unusual Perceptual Experiences and Odd Speech are not associated with phoneme or word accuracy in audiovisual speech.

Table 1

Correlations between target phoneme and word accuracy and Unusual Perceptual Experiences and Odd Speech subscales for audiovisual speech.

$r(97) \quad 95 \% \mathrm{CI} \quad p$-value $\quad$ Bayes

Factor

Unusual Perceptual Experiences

Phoneme Accuracy

.071

$[-0.128,0.265]$

.483

0.160

Word Accuracy

.023

$[-0.175,0.219]$

.822

0.129

\section{Odd Speech}

Phoneme Accuracy

$-.009 \quad[-0.206,0.189]$

.933

0.126

Word Accuracy

$-.021 \quad[-0.217,0.177]$

.833

0.128 
Multisensory gain, as measured by absolute increase from predicted audiovisual accuracy to actual audiovisual accuracy, was not significantly correlated with scores on the Unusual Perceptual Experiences subscale for phoneme accuracy or for word accuracy. Multisensory gain measured by the proportion increase from predicted audiovisual accuracy to actual audiovisual accuracy was also not significantly correlated with scores on the Unusual Perceptual Experiences subscale for phoneme accuracy or for word accuracy. Likewise, multisensory gain measured by absolute increase was not significantly correlated with scores on the Odd Speech subscale for phoneme accuracy or for word accuracy. Multisensory gain measured by proportion increase was not significantly correlated with scores on the Odd Speech subscale for phoneme accuracy or for word accuracy (Table 2). As all Bayes factors were below 0.144, this provides substantial evidence (according to Jeffreys in Jarosz \& Wiley (2014)) in support of the null hypothesis that Unusual Perceptual Experiences and Odd Speech are not associated with multisensory gain for audiovisual speech. 
Table 2

Correlations between multisensory gain and Unusual Perceptual Experiences and Odd Speech subscales for audiovisual speech.

$r(97) \quad 95 \% \mathrm{CI} \quad$-value $\quad$ Bayes

Factor

\section{Unusual Perceptual Experiences}

Phoneme Accuracy

$\begin{array}{lllll}\text { Absolute Increase } & -.053 & {[-0.248,0.146]} & .600 & 0.144 \\ \text { Proportion Increase } & -.025 & {[-0.221,0.173]} & .804 & 0.130\end{array}$

Word Accuracy

$\begin{array}{llccc}\text { Absolute Increase } & -.039 & {[-0.235,0.160]} & .699 & 0.135 \\ \text { Proportion Increase } & -.019 & {[-0.216,0.179]} & .850 & 0.128\end{array}$

\section{Odd Speech}

Phoneme Accuracy

$\begin{array}{llccc}\text { Absolute Increase } & .000 & {[-0.197,0.197]} & .997 & 0.126 \\ \text { Proportion Increase } & -.007 & {[-0.204,0.191]} & .946 & 0.126\end{array}$

Word Accuracy

$\begin{array}{lcccc}\text { Absolute Increase } & -.051 & {[-0.246,0.148]} & .616 & 0.142 \\ \text { Proportion Increase } & -.030 & {[-0.226,0.168]} & .768 & 0.131\end{array}$

In the audiovisual Same Time condition, distractor phoneme susceptibility was not significantly correlated with scores on the Unusual Perceptual Experiences subscale, nor was distractor word susceptibility. Similarly, in the audiovisual Same Time condition, distractor 
phoneme susceptibility was not significantly correlated with scores on the Odd Speech subscale, nor was distractor word susceptibility.

In the audiovisual Different Time condition, distractor phoneme susceptibility was not significantly correlated with scores on the Unusual Perceptual Experiences subscale, but distractor word susceptibility was significantly negatively correlated (Figure 4). In the audiovisual Different Time condition, distractor phoneme susceptibility was not significantly correlated with scores on the Odd Speech subscale, nor was distractor word susceptibility.

In the audiovisual Different Volume condition, distractor phoneme susceptibility was not significantly correlated with scores on the Unusual Perceptual Experiences subscale, nor was distractor word susceptibility. Similarly, in the audiovisual Different Volume condition, distractor phoneme susceptibility was not significantly correlated with scores on the Odd Speech subscale, nor was distractor word susceptibility (Table 3).

Almost all Bayes factors in this analysis were below 0.247, providing substantial evidence (according to Jeffreys in Jarosz \& Wiley (2014)) in support of the null hypothesis that Unusual Perceptual Experiences and Odd Speech are not associated with phoneme or word susceptibility for all conditions of the speech-in-noise task. The Bayes factor for the relationship between Unusual Perceptual Experiences and distractor word susceptibility in the Different Time condition provided weak or anecdotal evidence in support of the null hypothesis. 
Table 3

Correlations between distractor phoneme and word susceptibility and Unusual Perceptual Experiences and Odd Speech subscales for audiovisual speech conditions.

$r(97) \quad 95 \% \mathrm{CI} \quad p$-value $\quad$ Bayes

factor

\section{Same Time}

Unusual Perceptual Experiences

Phoneme Susceptibility

$-.068 \quad[-0.262,0.131]$

.502

0.157

Word Susceptibility

$-.031 \quad[-0.227,0.167]$

.762

0.131

Odd Speech

Phoneme Susceptibility

$.033 \quad[-0.166,0.229]$

.745

0.132

Word Susceptibility

$.054 \quad[-0.145,0.249]$

.597

0.144

\section{Different Time}

Unusual Perceptual Experiences

Phoneme Susceptibility

$-.115 \quad[-0.305,0.084]$

.258

0.236

Word Susceptibility

$-.203 \quad[-0.385,-0.006]$

.044

0.923

Odd Speech

Phoneme Susceptibility

$-.004 \quad[-0.201,0.194]$

.972

0.126

Word Susceptibility

$.000 \quad[-0.197,0.197]$

.998

0.126

\section{Different Volume}

Unusual Perceptual Experiences

Phoneme Susceptibility

$\begin{array}{lll}-.036 & {[-0.232,0.163]} & .724\end{array}$

0.134

Word Susceptibility

$-.119 \quad[-0.309,0.080]$

.242

0.247

\section{Odd Speech}

Phoneme Susceptibility

$.056 \quad[-0.143,0.251]$

.585

0.146

Word Susceptibility

$.082 \quad[-0.117,0.275]$

.417

0.174 


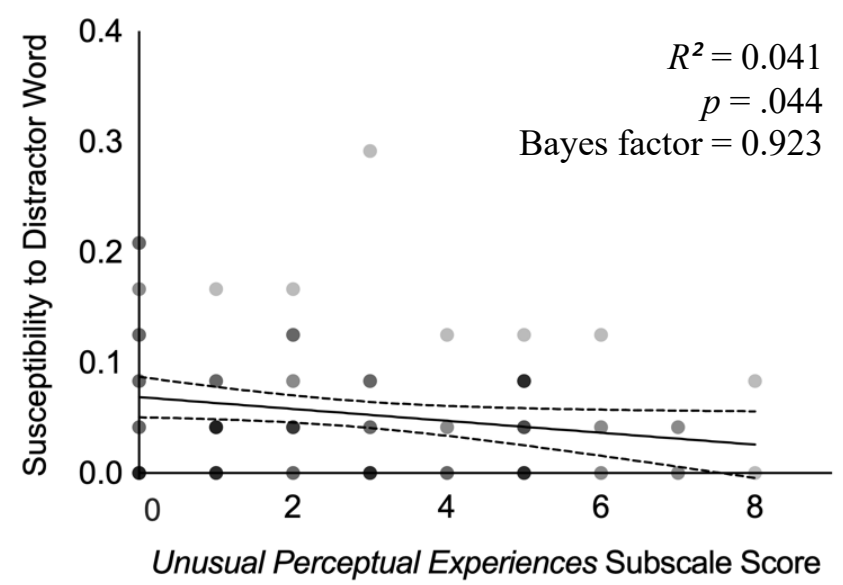

Figure 4. Scatterplot showing the correlation between Unusual Perceptual Experiences subscale score and susceptibility to the distractor word in the audiovisual Different Time condition of the speech-in-noise task $(N=99)$.

Note. Darker points indicate more individual points in that location.

\subsection{Discussion}

In Experiment 1, the relationship between performance in the speech-in-noise task and schizotypal traits, particularly Unusual Perceptual Experiences and Odd Speech, was investigated. No evidence was found for any relationship, and Bayesian analysis provided substantial evidence for the null in most cases. This included null findings in three a priori analyses comparing the Unusual Perceptual Experiences and Odd Speech subscales with audiovisual speech perception accuracy, multisensory gain, and susceptibility to distracting auditory speech in any condition (Same Time, Different Time, and Different Volume).

\section{Experiment 2}

\subsection{Rationale and Hypotheses}

In Experiment 2, the McGurk effect (McGurk \& MacDonald, 1976) was used as a measure of multisensory integration. In this task, participants were presented with a speaker uttering the auditory syllable "ba" coincidentally with the speaker visually articulating the 
syllable "ga". Participants commonly report perceiving the speaker saying "da" or "tha", a syllable that is not present in either of the unisensory stimuli, and a percept that is thus strong evidence of integration.

It was hypothesized that higher levels of schizotypal traits, specifically Unusual Perceptual Experiences and Odd Speech, would be associated with increased perception of the McGurk effect. While poorer multisensory integration would normally result in decreased perception of the McGurk effect, previous unpublished work from our lab revealed the opposite direction of results.

\subsection{Materials and Methods}

\subsubsection{Stimuli}

The videos used for the McGurk task have been used previously in studies of the McGurk effect (Quinto, Thompson, Russo, \& Trehub, 2010; Stevenson, Siemann, et al., 2014). Stimuli consisted of one audiovisual clip of a female speaker saying either the syllable "ba" or "ga", at a normal rate and volume with a neutral facial expression. Auditory stimuli were delivered at a comfortable level (calibrated to approximately $72 \mathrm{~dB}$ SPL) presented through noise-cancelling headphones. Visual stimuli were cropped to square, down-sampled to a resolution of $(400 \times 400$ pixels) spanning $11.8 \mathrm{~cm}$ per side or 11.23 degrees of visual angle and converted from color to grayscale. Presentations were shortened to $2 \mathrm{~s}$, and each presentation included the entire articulation of the syllable, including pre-articulatory gestures.

Stimuli included visual-only, auditory-only, and congruent audiovisual presentations of the phoneme "ba" or "ga," and the McGurk stimuli, a visual "ga" presented with an auditory "ba". All presentations were temporally synchronous.

\subsubsection{Procedures}


The task was divided into two separate runs, a multisensory and a unisensory run, always presented in that order. Both runs began with a screen instructing them to identify what syllable the speaker said in modality-neutral wording (“What did she say?”). Each trial began with a fixation screen that randomly jittered from 0.5 to 1.5 seconds, and multi-speaker babble which ramped up linearly for $500 \mathrm{~ms}$, continued during the stimulus presentation, and linearly ramped down over $500 \mathrm{~ms}$ following stimulus presentation. A $250 \mathrm{~ms}$ fixation screen was then presented, folled by a response screen asking "What did she say?". Participants responded via button press of, "b," "g," “d," or “t," representing "ba," "ga," “da," and "tha," respectively. Immediately after the response, the fixation screen for the subsequent trial was presented.

Audiovisual conditions included congruent "ba", congruent "ga", and the McGurk stimulus, an auditory "ba" paired with a "ga". Each audiovisual condition presented 20 times in random order, for a total of 60 trials. Unisensory conditions included auditory- and visual only presentations of "ba" and "ga". Each unisensory condition was presented 10 times in random order, for a total of 40 trials.

\subsubsection{Analysis}

For each of the six non-McGurk conditions, an accuracy score was calculated for each participant. This was calculated as the proportion of trials the participant accurately identified as the syllable that was presented. Average visual and auditory accuracy was calculated as the average proportion of visual- and auditory-alone trials perceived correctly across syllables.

For the McGurk trials, the proportion of McGurk percept was initially calculated as the proportion of trials the participant reported having perceived "da" or "tha". To account for some individuals" increased reporting of "da" or "tha" in the absence of the illusion, the absolute change from unisensory to multisensory reports of "da" or "tha" was calculated: 


$$
p(A V M c G u r k)-p(A+V-(A * V))
$$

where $\mathrm{p}(\mathrm{AV}$ McGurk) represents the individual's proportion of McGurk percepts with audiovisual McGurk stimuli, and $\mathrm{p}(\mathrm{A}+\mathrm{V}-(\mathrm{A} * \mathrm{~V}))$ represents the proportion of "da" percepts with unisensory "ba" and "ga" stimuli.

Pearson correlations were conducted between both McGurk perception rate and SPQ scores, and the absolute change and SPQ scores.

\subsection{Results}

The proportion of phonemes perceived are shown for each individual in Figure 5.

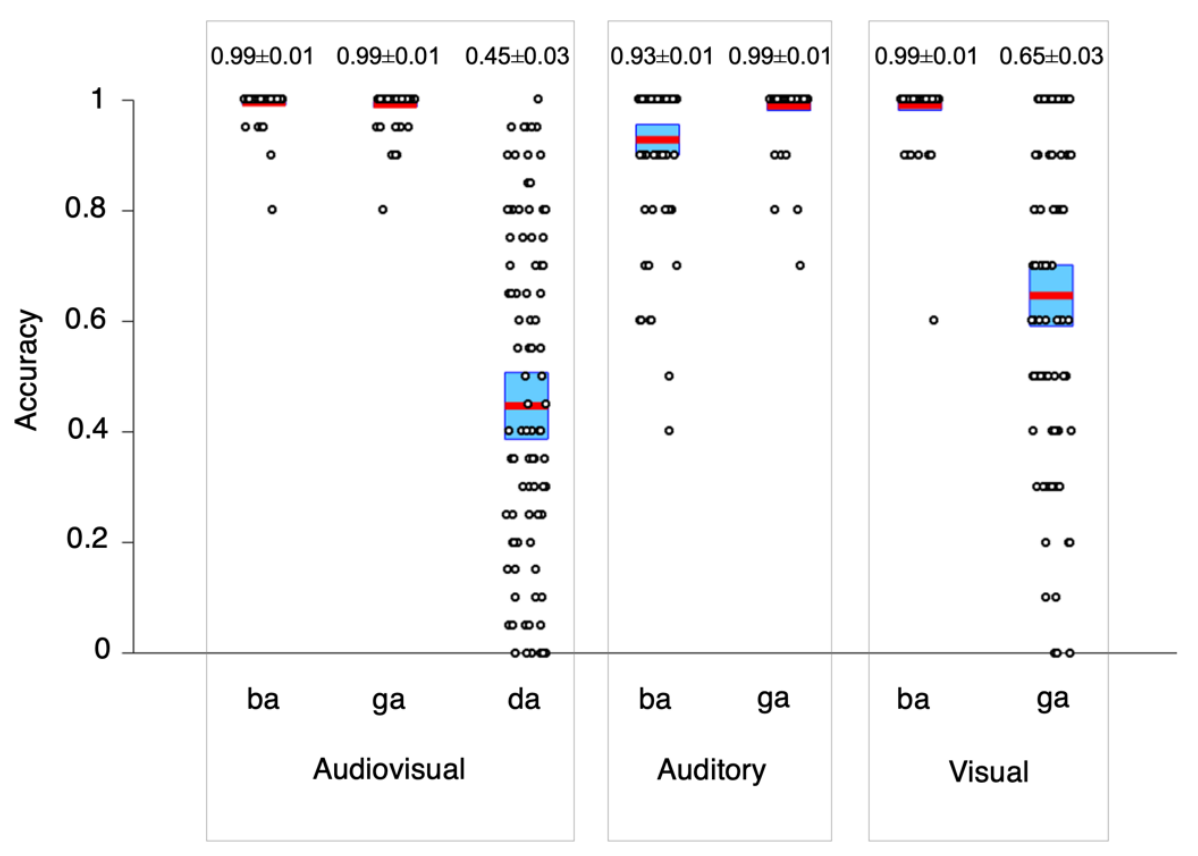

Figure 5. Proportion of phonemes perceived for congruent audiovisual "ba" and "ga", incongruent audiovisual "da" or McGurk effect, unisensory auditory "ba" and "ga", and unisensory visual "ba" and "ga" presentations.

Note. Red lines indicate group means, and blue indicates standard error. Accuracy represents the proportion of accurate responses. Values above each cluster indicate mean \pm standard error.

\subsubsection{Relating McGurk Task and Schizotypal Traits}


The proportion of trials on which the McGurk effect was perceived was expected a priori to negatively correlate with two subscales of the SPQ: Unusual Perceptual Experiences and Odd Speech. Surprisingly, perceived McGurk effect was not significantly correlated with scores on the Unusual Perceptual Experiences subscale, $r(97)=-.014,95 \% \mathrm{CI}=[-0.211,0.184], p=.887$, Bayes Factor $=0.127$, or the Odd Speech subscale, $r(97)=.028,95 \% \mathrm{CI}=[-0.170,0.224], p=$ .784 , Bayes Factor $=0.130$. As all Bayes factors were below 0.130, this provides substantial evidence (according to Jeffreys in Jarosz \& Wiley (2014)) in support of the null hypothesis that Unusual Perceptual Experiences and Odd Speech are not associated with the McGurk effect.

\subsection{Discussion}

Neither of the a priori subscales (Unusual Perceptual Experiences and Odd Speech) were related to perception of the McGurk effect. As the McGurk effect is a measure of multisensory integration, this suggests that the schizotypal traits measured in this sample are not related to this measure of multisensory integration.

\section{Experiment 3}

\subsection{Rationale and Hypotheses}

In Experiment 3, a speech-based ternary synchrony judgment task (SJ3; Alcalá-Quintana \& García-Pérez, 2013) was used to measure temporal processing. From this SJ3 task, we measured temporal binding window and point of subjective simultaneity for each participant. The PSS is the timepoint at which an individual determines two stimuli to be synchronized. It was hypothesized that higher levels of schizotypal traits, specifically Unusual Perceptual Experiences and Odd Speech, would be associated with both larger temporal binding window and point of subjective simultaneity.

\subsection{Materials and Methods}




\subsubsection{Stimuli}

Stimuli for the ternary synchrony judgment (SJ3) task were identical to the McGurk task, except that only the "ba" audiovisual stimuli were included. As in the McGurk task, stimuli included multi-speaker babble. Stimulus onset asynchronies (SOAs) were $0, \pm 50, \pm 100, \pm 150$, $\pm 200, \pm 300$, and $\pm 400 \mathrm{~ms}$, with positive values denoting a presentation with a visual lead and negative values an auditory lead. An SOA of zero denotes a synchronous presentation.

\subsubsection{Procedures}

Each SOA was presented randomly 10 times, with a total of 130 audiovisual presentations of the syllable. After each presentation, the response screen gave three options: $1=$ visual first, 2 = same time, or $3=$ audio first. Following participant response, a fixation cross was presented for $500 \mathrm{~ms}$ plus a randomly generated jitter drawn from a standard uniform distribution from $0-1000 \mathrm{~ms}$, followed by the initiation of the next stimulus presentation.

\subsubsection{Analysis}

Responses from the SJ3 task were used to calculate a temporal binding window and point of subjective simultaneity for each participant. The MATLAB analysis protocol from (AlcaláQuintana \& García-Pérez, 2013) was used. First, the count of responses for each SOA was calculated. This is the number of trials for a given SOA in which the individual responded with "audio first", "visual first", or "synchronous". The three response-types for each SOA were then fitted to three psychometric functions. Two sigmoid curves were fitted to the audio-first and visual-first responses, and one parabolic curve was fitted to the synchronous responses at each SOA. The audio-first simultaneity boundary was calculated as the crossing point of the psychometric functions for audio-first and synchronous judgments. The visual-first simultaneity boundary was calculated as the crossing point of the psychometric functions for visual-first and 
synchronous judgments. The temporal binding window was calculated as the distance between the audio-first and visual-first simultaneity boundaries. The point of subjective simultaneity was calculated as the midway point between the two simultaneity boundaries, or the peak of the parabolic curve for synchronous responses (Alcalá-Quintana \& García-Pérez, 2013).

Pearson correlations were then conducted between the temporal binding window and scores on the Unusual Perceptual Experiences and Odd Speech subscales of the SPQ, as well as between the point of subjective simultaneity and scores on these subscales.

\subsection{Results}

Temporal binding windows and point of subjective simultaneity are shown for each individual in Figure 6.
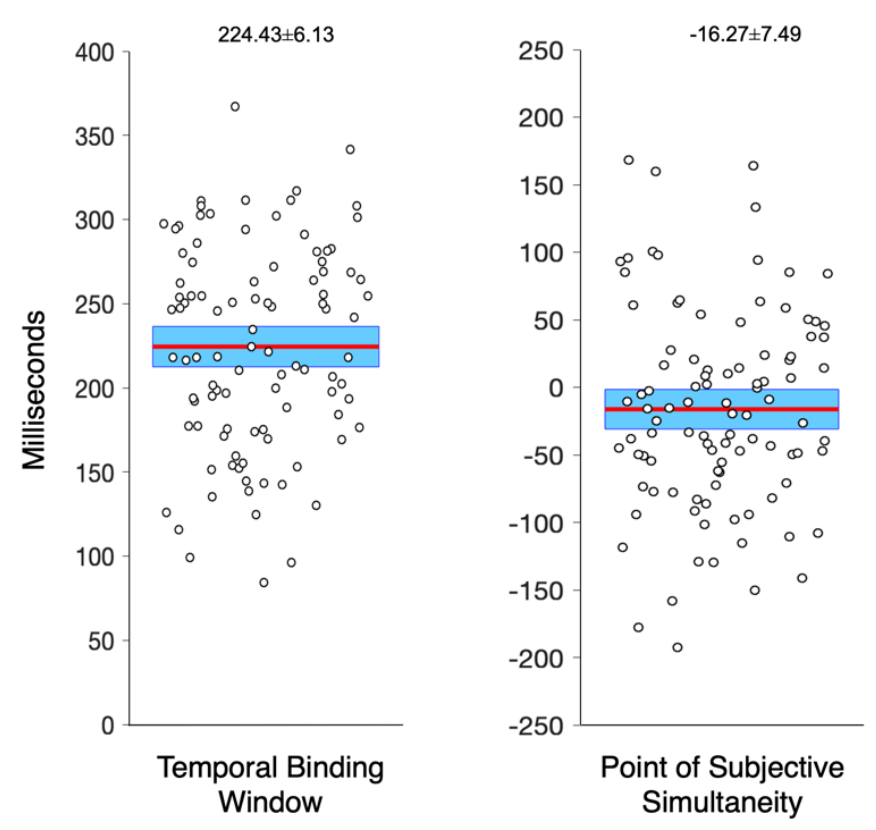

Figure 6. Temporal binding window and point of subjective simultaneity in milliseconds.

Note. Red lines indicate group means, and blue indicates standard error. Values above each cluster indicate mean \pm standard error.

\subsubsection{Relating Speech Ternary Synchrony Judgment Task and Schizotypal Traits}


Pearson correlations were conducted between temporal binding window and SPQ, and point of subjective simultaneity and SPQ scores. Temporal binding window and point of subjective simultaneity were expected a priori to positively correlate with Unusual Perceptual Experiences and Odd Speech. Neither temporal binding window nor point of subjective simultaneity were significantly correlated with either subscale (Table 4). As all Bayes factors were below 0.324 , this provides substantial evidence (according to Jeffreys in Jarosz \& Wiley (2014)) in support of the null hypothesis that Unusual Perceptual Experiences and Odd Speech are not associated with the temporal binding window or point of subjective simultaneity.

Table 4

Correlations between temporal binding window and point of subjective simultaneity and Unusual Perceptual Experiences and Odd Speech subscales.

\begin{tabular}{lcccc}
\hline & $r(97)$ & $95 \%$ CI & $p$-value & Bayes \\
& & & & Factor \\
\hline Unusual Perceptual Experiences & & & & \\
Temporal Binding Window & .031 & {$[-0.167,0.227]$} & .763 & .131 \\
Point of Subjective Simultaneity & -.066 & {$[-0.260,0.133]$} & .519 & .154 \\
Odd Speech & & & & \\
Temporal Binding Window & -.067 & {$[-0.261,0.132]$} & .508 & .156 \\
Point of Subjective Simultaneity & -.140 & {$[-0.328,0.059]$} & .165 & .324 \\
\hline
\end{tabular}

\subsection{Discussion}

We found no evidence that multisensory temporal processing was related to schizotypal traits in the general population. While Unusual Perceptual Experiences and Odd Speech were $a$ 
priori expected to correlate with temporal binding window and point of subjective simultaneity, neither were found to be related with these subscales.

\section{General Discussion}

This study investigated the relationship between perceptual- and speech-related schizotypal traits and measures of audiovisual speech perception, multisensory integration, and temporal processing. We hypothesized that individuals in the general population with higher levels of Unusual Perceptual Experiences and Odd Speech schizotypal traits would demonstrate reduced audiovisual speech perception, increased susceptibility to distracting auditory speech, lower multisensory integration, and altered multisensory temporal processing. We found evidence to the contrary, however. Individuals' levels of Unusual Perceptual Experiences and Odd Speech did not relate to audiovisual speech perception, as measured by a speech-in-noise task. Overall, these individuals also did not differ in the amount of susceptibility to distracting auditory speech in the speech-in-noise task, although an inconclusive relationship between Unusual Perceptual Experiences and distractor word susceptibility in the Different Time condition emerged. Individuals with higher levels of Unusual Perceptual Experiences and Odd Speech also did not differ in the amount of gain experienced from multisensory rather than unisensory speech, indicating no differences in multisensory integration. As another indicator of intact multisensory integration, these individuals did not show differences in the McGurk effect. Finally, levels of these traits were not related to differences in temporal processing, as measured by a speech-based ternary synchrony judgment task.

Our finding that audiovisual speech perception is not related to schizotypal traits is not in alignment with previous literature, in which individuals with schizophrenia have impaired auditory speech perception in the context of background speech noise (Hoffman et al., 1999; 
Shedlack et al., 1997; Wu et al., 2012), particularly individuals with schizophrenia who experience auditory hallucinations (Hoffman et al., 1999; Lee et al., 2004). Specifically, background speech results in greater impairments in speech perception than background white noise (Wu et al., 2012). However, individuals with schizophrenia are also impaired in the perception of auditory words without background noise (Bull \& Venables, 1974; DeLisi et al., 1997; Shedlack et al., 1997; Titone \& Levy, 2004). These deficits appear to be present at early stages of auditory phoneme processing, as revealed by altered event-related potentials in response to phoneme changes in individuals with schizophrenia (Kasai et al., 2002, 2003).

Similarly, our finding that schizotypal traits are not related to susceptibility to distracting auditory speech is surprising, considering previous findings in individuals within the schizophrenia spectrum. Individuals with schizophrenia are impaired at ignoring distracting speech, resulting in poorer perception accuracy of target speech and increased perception of irrelevant, distracting speech (Moser et al., 2001; Oltmanns \& Neale, 1975). Higher severity of disorganized speech is related to greater impairments in ignoring distracting speech (Moser et al., 2001). Additionally, a greater tendency to extract meaningful speech from incomprehensible overlapping background babble among individuals with prodromal psychosis symptoms is predictive of subsequent schizophrenia diagnosis (Hoffman et al., 2007). Individuals with higher levels of schizotypal traits are also more easily distracted by auditory speech (Marsh et al., 2017) and visual non-speech stimuli (Braunstein-Bercovitz \& Lubow, 1998). This greater susceptibility of individuals across the schizophrenia spectrum to attend to distracting auditory speech appears to be due to deficits either in the allocation of attention, in the available attentional resources (Bestelmeyer, 2012), or in sensory gating abilities (Mcdowd et al., 1993). 
Additionally, our findings that multisensory integration was not related to schizotypal traits in these individuals does not align with much of the related literature. Audiovisual multisensory integration has been previously investigated in relation to schizotypal traits in the general population, with increased integration being found in the form of stronger responses to the McGurk illusion (Muller, Dalal, \& Stevenson, 2020) and the double-flash illusion (Ferri, Venskus, Fotia, Cooke, \& Romei, 2018). Investigations using visual-tactile illusions, which require multisensory integration, have also been conducted. Individuals with higher schizotypy tend to have stronger responses to visual-tactile (rubber-hand and Barbie doll) illusions (Asai et al., 2011; Germine et al., 2013; Van Doorn et al., 2018), which also aligns with findings in schizophrenia (Peled, Ritsner, Hirschmann, Geva, \& Modai, 2000; Thakkar, Nichols, Mcintosh, $\&$ Park, 2011). These illusions, while requiring intact multisensory integration, also assess traits like susceptibility to out-of-body experiences and altered perception of body ownership, which are higher among individuals in the schizophrenia spectrum (Benson, Brugger, \& Park, 2019; Hur, Kwon, Lee, \& Park, 2014). This may explain why the intact multisensory integration found in these visual-tactile illusions does not align with the majority of investigations into audiovisual multisensory integration in individuals with schizophrenia, which have found decreased multisensory integration compared to controls (de Jong, Hodiamont, Van den Stock, \& de Gelder, 2009; Ross et al., 2007; Tseng et al., 2015; Williams, Light, Braff, \& Ramachandran, 2010). Deficits in multisensory integration in schizophrenia are found especially in the realm of audiovisual speech integration (Tseng et al., 2015). While there have been some findings of intact multisensory integration in schizophrenia (e.g. de Boer-Schellekens et al., 2014), in general this is not the case (for a review see Zhou et al., 2018). 
Our finding that individuals with higher levels of perceptual- and speech-related schizotypal traits demonstrated intact temporal processing is also inconsistent with previous literature in this area. Albeit scarce, this literature includes findings that individuals in the general population with higher levels of schizotypal traits have poorer temporal processing in tactile-proprioceptive (Ferri et al., 2016) and audio-tactile (Ferri et al., 2017) domains, suggesting wider temporal binding windows. Additionally, those with higher schizotypy have also been found to have increased perception of the audiovisual double-flash (fission) illusion (Ferri, Venskus, Fotia, \& Cooke, 2018), which also suggests wider temporal binding windows in these individuals. Similarly, numerous findings have indicated impaired temporal processing and wider temporal binding windows in individuals with schizophrenia, both in the visual modality alone (Capa et al., 2014; de Boer-Schellekens et al., 2014; Giersch et al., 2009; Lalanne et al., 2012; Schmidt et al., 2011; Tenckhoff et al., 2002) and in audiovisual modalities together (Foucher et al., 2007; Martin et al., 2013; Noel et al., 2018; Stevenson, Park, et al., 2017). While Stevenson et al. (2017) found that individuals with schizophrenia had less precise temporal processing than controls, they found that within individuals with schizophrenia, those who had more severe hallucinations tended to have narrower temporal binding windows. Together, these findings suggest that there is a complex relationship between temporal processing and schizophrenia symptoms.

These null findings provide a number of interesting insights towards understanding multisensory speech perception in the schizophrenia spectrum. One possibility is that the perceptual differences observed in schizophrenia are specific to individuals diagnosed with schizophrenia diagnoses, and that individuals in the general population with subclinical schizotypal traits may not show these perceptual differences. That is, there may be a non-linear 
relationship between level of severity on the schizophrenia spectrum and degree of perceptual differences such that the relationship is weak or non-existent at lower levels of schizotypy, but becomes more substantive as clinical severity increases. It is also possible that the schizophrenia spectrum may be better described by a quasi-dimensional view rather than a fully dimensional view.

Additionally, there may be more experiment-based explanations for finding these null effects in the context of disagreeing literature. Males tend to score higher in schizotypy than females (Bora \& Baysan Arabaci, 2009), and male risk for psychosis peaks between ages 21 and 25 (Kessler et al., 2007; Li, Ma, Yang, \& Wang, 2016). The peak age of psychosis onset in females is three to five years later than males, ranging from ages 25 to 30 (Li et al., 2016). Our sample, however, had a mean age of 18 and a high proportion of female participants $(>70 \%)$. As a result, it is possible that the levels of schizotypal traits in the current sample were simply too low to be able to detect perceptual alterations, though scores on the SPQ did span a significant portion of the range. Likewise, given the sex differences present in schizotypal traits and onset of psychosis, it is also possible that there are sex differences in perceptual symptoms. It may thus be fruitful for future work to investigate schizotypal traits in a slightly older, less female-dominated, community sample. Likewise, it may be beneficial to use multiple measures of schizotypy to increase our ability to reliably detect schizotypal traits in this sample.

\subsection{Conclusion}

To conclude, the current study did not find evidence of a relationship between schizotypal traits and altered multisensory integration, audiovisual temporal processing, audiovisual speech perception, or auditory distractibility. Considering that the literature in this area includes several findings of perceptual differences among individuals in the schizophrenia 
spectrum, this area certainly requires further investigation to elucidate the nature of these findings.

\section{CRediT authorship contribution statement}

Anne-Marie Muller: Conceptualization, Methodology, Software, Formal analysis, Investigation, Data curation, Writing - Original Draft, Writing - Review and Editing, Visualization. Tyler Dalal: Investigation, Writing - Review and Editing. Ryan Stevenson:

Conceptualization, Methodology, Software, Writing - Reviewing and Editing, Supervision, Project administration, Funding acquisition.

\section{Acknowledgements}

RS is funded by an NSERC Discovery Grant (RGPIN-2017-04656), a SSHRC Insight Grant (R5502A07), the University of Western Ontario Faculty Development Research Fund, the province of Ontario Early Researcher Award, and a Canadian Foundation for Innovation John R. Evans Leaders Fund (37497). AM is funded by a Canada Graduate Scholarship from the Canadian Institutes of Health Research.

\section{References}

Alcalá-Quintana, R., \& García-Pérez, M. A. (2013). Fitting model-based psychometric functions to simultaneity and temporal-order judgment data: MATLAB and R routines. Behavioral Research, 45, 972-998. https://doi.org/10.3758/s13428-013-0325-2

Asai, T., Mao, Z., Sugimori, E., \& Tanno, Y. (2011). Rubber hand illusion, empathy, and schizotypal experiences in terms of self-other representations. Consciousness and Cognition, 20, 1744-1750. https://doi.org/10.1016/j.concog.2011.02.005

Badcock, J. C., \& Dragović, M. (2006). Schizotypal personality in mature adults. Personality and Individual Differences, 40, 77-85. https://doi.org/10.1016/j.paid.2005.06.015 
Benson, T. L., Brugger, P., \& Park, S. (2019). Bodily self-disturbance in schizophrenia-spectrum populations: Introducing the Benson et al. Body Disturbances Inventory (B-BODI). PsyCh Journal, 8, 110-121. https://doi.org/10.1002/pchj.280

Bestelmeyer, P. E. G. (2012). The visual P3a in schizophrenia and bipolar disorder: Effects of target and distractor stimuli on the P300. Psychiatry Research, 197, 140-144. https://doi.org/10.1016/j.psychres.2011.09.030

Bora, E., \& Baysan Arabaci, L. (2009). Effect of age and gender on schizotypal personality traits in the normal population. Psychiatry and Clinical Neurosciences, 63, 663-669. https://doi.org/10.1111/j.1440-1819.2009.02011.x

Brainard, D. H. (1997). The Psychophysics Toolbox. Spatial Vision, 10, 433-436. https://doi.org/10.1163/156856897X00357

Braunstein-Bercovitz, H., \& Lubow, R. E. (1998). Are high-schizotypal normal participants distractible or limited in attentional resources? A study of latent inhibition as a function of masking task load and schizotypy level. Journal of Abnormal Psychology, 107, 659-670. https://doi.org/10.1037/0021-843X.107.4.659

Bull, H. C., \& Venables, P. H. (1974). Speech perception in schizophrenia. British Journal of Psychiatry, 125, 350-354. https://doi.org/10.1192/bjp.125.4.350

Capa, R. L., Duval, C. Z., Blaison, D., \& Giersch, A. (2014). Patients with schizophrenia selectively impaired in temporal order judgments. Schizophrenia Research, 156, 51-55. https://doi.org/10.1016/j.schres.2014.04.001

Chen, W. J., Hsiao, C. K., \& Lin, C. C. H. (1997). Schizotypy in community samples: The threefactor structure and correlation with sustained attention. Journal of Abnormal Psychology, 106, 649-654. https://doi.org/10.1037/0021-843X.106.4.649 
de Boer-Schellekens, L., Stekelenburg, J. J., Maes, J. P., Van Gool, A. R., \& Vroomen, J. (2014). Sound improves diminished visual temporal sensitivity in schizophrenia. Acta Psychologica, 147, 136-142. https://doi.org/10.1016/j.actpsy.2013.06.013

de Jong, J. J., Hodiamont, P. P. G., Van den Stock, J., \& de Gelder, B. (2009). Audiovisual emotion recognition in schizophrenia: Reduced integration of facial and vocal affect. Schizophrenia Research, 107, 286-293. https://doi.org/10.1016/j.schres.2008.10.001

DeLisi, L. E., Sakuma, M., Kushner, M., Finer, D. L., Hoff, A. L., \& Crow, T. J. (1997). Anomalous cerebral asymmetry and language processing in schizophrenia. Schizophrenia Bulletin, 23, 255-271. https://doi.org/10.1093/schbul/23.2.255

Ettinger, U., Meyhöfer, I., Steffens, M., Wagner, M., \& Koutsouleris, N. (2014). Genetics, cognition, and neurobiology of schizotypal personality: a review of the overlap with schizophrenia. Frontiers in Psychiatry, 5, 1-16. https://doi.org/10.3389/fpsyt.2014.00018

Ferri, F., Ambrosini, E., \& Costantini, M. (2016). Spatiotemporal processing of somatosensory stimuli in schizotypy. Scientific Reports, 6(38735), 1-8. https://doi.org/10.1038/srep38735

Ferri, F., Nikolova, Y. S., Perrucci, M. G., Costantini, M., Ferretti, A., Gatta, V., ... Northoff, G. (2017). A Neural "Tuning Curve" for Multisensory Experience and Cognitive-Perceptual Schizotypy. Schizophrenia Bulletin, 43, 801-813. https://doi.org/10.1093/schbul/sbw174

Ferri, F., Venskus, A., Fotia, F., \& Cooke, J. (2018). Higher proneness to multisensory illusions is driven by reduced temporal sensitivity in people with high schizotypal traits. Consciousness and Cognition, 65, 263-270. https://doi.org/10.1016/j.concog.2018.09.006

Ferri, F., Venskus, A., Fotia, F., Cooke, J., \& Romei, V. (2018). Higher proneness to multisensory illusions is driven by reduced temporal sensitivity in people with high schizotypal traits. Consciousness and Cognition, 65, 263-270. 
https://doi.org/10.1016/j.concog.2018.09.006

Fossati, A., Raine, A., Carretta, I., Leonardi, B., \& Maffei, C. (2003). The three-factor model of schizotypal personality: invariance across age and gender. Personality and Individual Differences, 35, 1007-1019. https://doi.org/10.1016/S0191-8869(02)00314-8

Foucher, J. R., Lacambre, M., Pham, B. T., Giersch, A., \& Elliott, M. A. (2007). Low time resolution in schizophrenia: Lengthened windows of simultaneity for visual, auditory and bimodal stimuli. Schizophrenia Research, 97, 118-127. https://doi.org/10.1016/j.schres.2007.08.013

Friston, K., Brown, H. R., Siemerkus, J., \& Stephan, K. E. (2016). The dysconnection hypothesis (2016). Schizophrenia Research, 176, 83-94. https://doi.org/10.1016/j.schres.2016.07.014

Germine, L., Leigh, T., Cohen, F., \& Lee, C. I. (2013). Psychosis-proneness and the rubber hand illusion of body ownership. Psychiatry Research, 207, 45-52. https://doi.org/10.1016/j.psychres.2012.11.022

Giersch, A., Lalanne, L., Corves, C., Seubert, J., Shi, Z., Foucher, J., \& Elliott, M. A. (2009). Extended visual simultaneity thresholds in patients with schizophrenia. Schizophrenia Bulletin, 35, 816-825. https://doi.org/10.1093/schbul/sbn016

Hoffman, R. E., Rapaport, J., Mazure, C. M., \& Quinlan, D. M. (1999). Selective Speech Perception Alterations in Schizophrenic Patients Reporting Hallucinated "Voices." American Journal of Psychiatry, 156, 393-399.

Hoffman, R. E., Woods, S. W., Hawkins, K. A., Pittman, B., Tohen, M., Preda, A., ... McGlashan, T. H. (2007). Extracting spurious messages from noise and risk of schizophrenia-spectrum disorders in a prodromal population. British Journal of Psychiatry, 191, 355-356. https://doi.org/10.1192/bjp.bp.106.031195 
Hur, J. W., Kwon, J. S., Lee, T. Y., \& Park, S. (2014). The crisis of minimal self-awareness in schizophrenia: A meta-analytic review. Schizophrenia Research, 152, 58-64. https://doi.org/10.1016/j.schres.2013.08.042

Javitt, D. C., Liederman, E., Cienfuegos, A., \& Shelley, A. M. (1999). Panmodal processing imprecision as a basis for dysfunction of transient memory storage systems in schizophrenia. Schizophrenia Bulletin, 25, 763-775. https://doi.org/10.1093/oxfordjournals.schbul.a033417

Jones, S. R. (2010). Do we need multiple models of auditory verbal hallucinations? Examining the phenomenological fit of cognitive and neurological models. Schizophrenia Bulletin, 36, 566-575. https://doi.org/10.1093/schbul/sbn129

Jones, S. R., \& Fernyhough, C. (2007). Thought as action: Inner speech, self-monitoring, and auditory verbal hallucinations. Consciousness and Cognition, 16, 391-399. https://doi.org/10.1016/j.concog.2005.12.003

Kasai, K., Nakagome, K., Itoh, K., Koshida, I., Hata, A., Iwanami, A., ... Kato, N. (2002). Impaired cortical network for preattentive detection of change in speech sounds in schizophrenia: A high-resolution event-related potential study. American Journal of Psychiatry, 159, 546-553. https://doi.org/10.1176/appi.ajp.159.4.546

Kasai, K., Yamada, H., Kamio, S., Nakagome, K., Iwanami, A., Fukuda, M., ... Kato, N. (2003). Neuromagnetic correlates of impaired automatic categorical perception of speech sounds in schizophrenia. Schizophrenia Research, 59, 159-172. https://doi.org/10.1016/S09209964(01)00382-6

Kessler, R. C., Amminger, G. P., Aguilar-Gaxiola, S., Alonso, J., Lee, S., \& Üstün, T. B. (2007). Age of onset of mental disorders: A review of recent literature. Current Opinion in 
Psychiatry, 20, 359-364. https://doi.org/10.1097/YCO.0b013e32816ebc8c

Kwapil, T. R., Gross, G. M., Silvia, P. J., \& Barrantes-Vidal, N. (2013). Prediction of psychopathology and functional impairment by positive and negative schizotypy in the Chapmans' ten-year longitudinal study. Journal of Abnormal Psychology, 122, 807-815. https://doi.org/10.1037/a0033759

Lalanne, L., Van Assche, M., Wang, W., \& Giersch, A. (2012). Looking forward: An impaired ability in patients with schizophrenia? Neuropsychologia, 50, 2736-2744. https://doi.org/10.1016/j.neuropsychologia.2012.07.023

Lee, S. H., Chung, Y. C., Yang, J. C., Kim, Y. K., \& Suh, K. Y. (2004). Abnormal speech perception in schizophrenia with auditory hallucinations. Acta Neuropsychiatrica, 16, 154159. https://doi.org/10.1111/j.0924-2708.2004.00071.x

Li, R., Ma, X., Yang, J., \& Wang, C. (2016). Why Sex Differences in Schizophrenia? Journal of Translational Neuroscience, 1, 37-42. https://doi.org/10.1001/archpsyc.1997.01830140101019

Lim, A., Hoek, H. W., Deen, M. L., Blom, J. D., Bruggeman, R., Cahn, W., ... Wiersma, D. (2016). Prevalence and classification of hallucinations in multiple sensory modalities in schizophrenia spectrum disorders. Schizophrenia Research, 176, 493-499. https://doi.org/10.1016/J.SCHRES.2016.06.010

Marsh, J. E., Vachon, F., \& Sörqvist, P. (2017). Increased distractibility in schizotypy: Independent of individual differences in working memory capacity? Quarterly Journal of Experimental Psychology, 70, 565-578. https://doi.org/10.1080/17470218.2016.1172094

Martin, B., Giersch, A., Huron, C., \& Wassenhove, V. Van. (2013). Temporal event structure and timing in schizophrenia: Preserved binding in a longer "“now." Neuropsychologia, 51, 
358-371. https://doi.org/10.1016/j.neuropsychologia.2012.07.002

McCarthy-Jones, S., Smailes, D., Corvin, A., Gill, M., Morris, D. W., Dinan, T. G., ... Dudley, R. (2017). Occurrence and co-occurrence of hallucinations by modality in schizophreniaspectrum disorders. Psychiatry Research, 252, 154-160. https://doi.org/10.1016/J.PSYCHRES.2017.01.102

Mcdowd, J. M., Filion, D. L., Harris, M. J., \& Braff, D. L. (1993). Sensory gating and inhibitory function in late-life schizophrenia. Schizophrenia Bulletin, 19, 733-746. https://doi.org/10.1093/schbul/19.4.733

McGurk, H., \& MacDonald, J. (1976). Hearing lips and seeing voices. Nature, 264, 746-748. https://doi.org/10.1038/264746a0

Moser, R. K., Cienfuegos, A., Barros, J., \& Javitt, D. (2001). Auditory distraction and thought disorder in chronic schizophrenic inpatients: Evidence for separate contributions by incapacity and poor allocation and a subsyndrome related to the allocation deficit. Schizophrenia Research, 51, 163-170. https://doi.org/10.1016/S0920-9964(00)00122-5

Muller, A.-M., Dalal, T. C., \& Stevenson, R. A. (2020). Schizotypal Personality Traits and Multisensory Integration: An Investigation Using the McGurk Effect. https://doi.org/https://doi.org/10.31234/osf.io/qrmuk

Noel, J. P., Stevenson, R. A., \& Wallace, M. T. (2018). Atypical audiovisual temporal function in autism and schizophrenia: Similar phenotype, different cause. European Journal of Neuroscience, 47, 1230-1241. https://doi.org/10.1111/ejn.13911

Oltmanns, T. F., \& Neale, J. M. (1975). Schizophrenic performance when distractors are present: Attentional deficit or differential task difficulty? Journal of Abnormal Psychology, 84, 205209. https://doi.org/10.1037/h0076721 
Peled, A., Ritsner, M., Hirschmann, S., Geva, A. B., \& Modai, I. (2000). Touch Feel Illusion in Schizophrenic Patients. Society of Biological Psychiatry, 48, 1105-1108.

Pelli, D. G. (1997). The VideoToolbox software for visual psychophysics: Transforming numbers into movies. Spatial Vision, 10, 437-442. https://doi.org/10.1163/156856897X00366

Quinto, L., Thompson, W. F., Russo, F. A., \& Trehub, S. E. (2010). A comparison of the McGurk effect for spoken and sung syllables. Attention, Perception, \& Psychophysics, 72, 1450-1454. https://doi.org/10.3758/APP

Raine, A. (1991). The SPQ: A scale for the assessment of schizotypal personality based on DSM-III-R criteria. Schizophrenia Bulletin, 17, 555-564. https://doi.org/10.1093/schbul/17.4.555

Raine, A., Lencz, T., Scerbo, A., \& Kim, D. (1994). Cognitive-Perceptual, Interpersonal, and Disorganized Features of Schizotypal Personality. Schizophrenia Bulletin, 20, 191-201.

Reynolds, C. A., Raine, A., Mellingen, K., Venables, P. H., \& Mednick, S. A. (2000). Threefactor model of schizotypal personality: Invariance across culture, gender, religious affiliation, family adversity, and psychopathology. Schizophrenia Bulletin, 26, 603-618. https://doi.org/10.1093/oxfordjournals.schbul.a033481

Ross, L. A., Saint-Amour, D., Leavitt, V. M., Javitt, D. C., \& Foxe, J. J. (2007). Do you see what I am saying? Exploring visual enhancement of speech comprehension in noisy environments. Cerebral Cortex, 17, 1147-1153. https://doi.org/10.1093/cercor/bhl024

Ross, L. A., Saint-Amour, D., Leavitt, V. M., Molholm, S., Javitt, D. C., \& Foxe, J. J. (2007). Impaired multisensory processing in schizophrenia: Deficits in the visual enhancement of speech comprehension under noisy environmental conditions. Schizophrenia Research, 97, 
173-183. https://doi.org/10.1016/j.schres.2007.08.008

Schmidt, H., McFarland, J., Ahmed, M., McDonald, C., \& Elliott, M. A. (2011). Low-level temporal coding impairments in psychosis: Preliminary findings and recommendations for further studies. Journal of Abnormal Psychology, 120, 476-482. https://doi.org/10.1037/a0023387

Schonauer, K., Achtergarde, D., \& Reker, T. (1998). Lipreading in prelingually deaf and hearing patients with schizophrenia. Journal of Nervous and Mental Disease, 186, 247-249. https://doi.org/10.1097/00005053-199804000-00008

Shedlack, K., Lee, G., Sakuma, M., Xie, S. H., Kushner, M., Pepple, J., ... DeLisi, L. E. (1997). Language processing and memory in ill and well siblings from multiplex families affected with schizophrenia. Schizophrenia Research, 25, 43-52. https://doi.org/10.1016/S09209964(97)00004-2

Stein, B. E., \& Meredith, M. A. (1993). The Merging of the Senses. The MIT Press. https://doi.org/10.1162/jocn.1993.5.3.373

Stevenson, R. A., Ghose, D., Fister, J. K., Sarko, D. K., Altieri, N. A., Nidiffer, A. R., ... Wallace, M. T. (2014). Identifying and Quantifying Multisensory Integration: A Tutorial Review. Brain Topography, 27, 707-730. https://doi.org/10.1007/s10548-014-0365-7

Stevenson, R. A., Nelms, C. E., Baum, S. H., Zurkovsky, L., Barense, M. D., Newhouse, P. A., \& Wallace, M. T. (2015). Deficits in audiovisual speech perception in normal aging emerge at the level of whole-word recognition. Neurobiology of Aging, 36, 283-291. https://doi.org/10.1016/j.neurobiolaging.2014.08.003

Stevenson, R. A., Park, S., Cochran, C., McIntosh, L. G., Noel, J. P., Barense, M. D., ... Wallace, M. T. (2017). The associations between multisensory temporal processing and 
symptoms of schizophrenia. Schizophrenia Research, 179, 97-103. https://doi.org/10.1016/j.schres.2016.09.035

Stevenson, R. A., Segers, M., Ncube, B. L., Black, K. R., Bebko, J. M., Ferber, S., \& Barense, M. D. (2017). The cascading influence of multisensory processing on speech perception in autism. Autism, 22, 609-624. https://doi.org/10.1177/1362361317704413

Stevenson, R. A., Siemann, J. K., Schneider, B. C., Eberly, H. E., Woynaroski, T. G., Camarata, S. M., \& Wallace, M. T. (2014). Multisensory temporal integration in autism spectrum disorders. The Journal of Neuroscience, 34, 691-697. https://doi.org/10.1523/JNEUROSCI.3615-13.2014

Tenckhoff, A., Tost, H., \& Braus, D. F. (2002). Altered perception of temporal relationships in schizophrenic psychoses. Nervenarzt, 73, 428-433. https://doi.org/10.1007/s00115-001$1254-3$

Thakkar, K. N., Nichols, H. S., Mcintosh, L. G., \& Park, S. (2011). Disturbances in body ownership in schizophrenia: Evidence from the rubber hand illusion and case study of a spontaneous out-of-body experience. PLoS ONE, 6, e27089.

https://doi.org/10.1371/journal.pone.0027089

Titone, D., \& Levy, D. L. (2004). Lexical competition and spoken word identification in schizophrenia. Schizophrenia Research, 68, 75-85. https://doi.org/10.1016/S09209964(03)00212-3

Tseng, H.-H., Bossong, M. G., Modinos, G., Chen, K.-M., Mcguire, P., \& Allen, P. (2015). A systematic review of multisensory cognitive-affective integration in schizophrenia. Neuroscience and Biobehavioral Reviews, 55, 444-452. https://doi.org/10.1016/j.neubiorev.2015.04.019 
Van Doorn, G., De Foe, A., Wood, A., Wagstaff, D., \& Hohwy, J. (2018). Down the rabbit hole: Assessing the influence of schizotypy on the experience of the Barbie Doll Illusion. Cognitive Neuropsychiatry, 23, 284-298. https://doi.org/10.1080/13546805.2018.1495623

Vroomen, J., \& Keetels, M. (2010). Perception of intersensory synchrony: A tutorial review. Attention, Perception, \& Psychophysics, 72, 871-884. https://doi.org/10.3758/APP

Williams, L. E., Light, G. A., Braff, D. L., \& Ramachandran, V. S. (2010). Reduced multisensory integration in patients with schizophrenia on a target detection task. Neuropsychologia, 48, 3128-3136. https://doi.org/10.1016/j.neuropsychologia.2010.06.028 Wu, C., Cao, S., Zhou, F., Wang, C., Wu, X., \& Li, L. (2012). Masking of speech in people with first-episode schizophrenia and people with chronic schizophrenia. Schizophrenia Research, 134, 33-41. https://doi.org/10.1016/j.schres.2011.09.019

Wuthrich, V. M., \& Bates, T. C. (2006). Confirmatory factor analysis of the three-factor structure of the Schizotypal Personality Questionnaire and Chapman Schizotypy Scales. Journal of Personality Assessment, 87, 292-304. https://doi.org/10.1207/s15327752jpa8703

Zhou, H., Cai, X., Weigl, M., Bang, P., Cheung, E. F. C., \& Chan, R. C. K. (2018). Multisensory temporal binding window in autism spectrum disorders and schizophrenia spectrum disorders: A systematic review and meta-analysis. Neuroscience and Biobehavioral Reviews, 86, 66-76. https://doi.org/10.1016/j.neubiorev.2017.12.013 